This item was submitted to Loughborough's Research Repository by the author.

Items in Figshare are protected by copyright, with all rights reserved, unless otherwise indicated.

\title{
Mastery of structured quantities like finger or dice patterns predict arithmetic performance
}

PLEASE CITE THE PUBLISHED VERSION

https://doi.org/10.1007/s10339-020-00994-4

PUBLISHER

Springer

VERSION

AM (Accepted Manuscript)

\section{PUBLISHER STATEMENT}

This is a post-peer-review, pre-copyedit version of an article published in Cognitive Processing. The final authenticated version is available online at: https://doi.org/10.1007/s10339-020-00994-4.

\section{LICENCE}

CC BY-NC-ND 4.0

\section{REPOSITORY RECORD}

Kreilinger, Isabella Luise, Stephanie Roesch, Korbinian Moeller, and Silvia Pixner. 2020. "Mastery of Structured Quantities Like Finger or Dice Patterns Predict Arithmetic Performance". Loughborough University. https://hdl.handle.net/2134/13606739.v1. 


\section{Mastery of structured quantities like finger or dice patterns predict arithmetic performance}

Isabella Luise Kreilinger ${ }^{1}$, Stephanie Roesch ${ }^{2,3,4}$, Korbinian Moeller $^{5,2,6,7}$ \& Silvia Pixner ${ }^{1}$

${ }^{1}$ Developmental Psychology, Department of Psychology and Medical Sciences, UMIT - Private University for Health Sciences, Medical Informatics and Technology, 6060 Hall in Tyrol, Austria

${ }^{2}$ Leibniz-Institut für Wissensmedien, 72076 Tuebingen, Germany

${ }^{3}$ Department of Psychology and Lead Research Network, University of Tuebingen

${ }^{4}$ Hector Research Institute of Education Sciences and Psychology, University of Tuebingen

${ }^{5}$ Centre for Mathematical Cognition, School of Science, Loughborough University, Loughborough, United Kingdom

${ }^{6}$ LEAD Graduate School and Research Network, University of Tuebingen, Tuebingen Germany

${ }^{7}$ Center for Individual Development and Adaptive Education of Children at Risk (IDeA), Frankfurt, Germany

contact: isabella.kreilinger@umit.at

Eduard-Wallnöfer Zentrum 1, 6060 Hall in Tirol 


\begin{abstract}
In the present study, we investigated whether structured quantities like finger or dice patterns are enumerated better than unstructured quantities because they may not require counting. Moreover, we hypothesized children's mastery of structured quantities to predict their later arithmetic performance longitudinally. In particular, we expected that children more proficient in enumerating structured quantities early in their numerical development, should develop more effective calculation strategies later because they may rely on counting less. Therefore, we conducted a longitudinal study (including 116 children, 58 girls) over the course of about 7 months from preschool (at about 6 years of age) to the middle of first grade. Results showed that structured quantities were indeed enumerated more accurately and faster than unstructured quantities in preschool. Additionally, we observed significant associations of enumeration of structured and unstructured with children's addition performance in first grade. However, regression analysis indicated only enumeration of structured but not unstructured quantities to significantly predict later addition performance. In sum, this longitudinal study clearly indicates that mastery of structured quantities seems to be beneficial for children's development of basic arithmetic abilities.
\end{abstract}

key words: basic numerical abilities, finger patterns, dice patterns, counting, arithmetic, preschoolers 


\section{Introduction}

The relevance of basic numerical abilities as the foundation of later more advanced numerical and mathematical skills is uncontroversial (e.g. Bishop 1989; Rivera-Batiz 1992; Starr et al. 2013; Pixner et al. 2017). Basic numerical abilities to capture quantities such as subitizing, counting, or enumeration skills have been shown repeatedly to predict children's further numerical development but also mathematics achievement in primary school (e.g., Geary 2000) and beyond (e.g., Hirsch et al. 2018). Most of these basic abilities for grasping numerical quantities develop during early childhood years before children receive formal mathematics instruction in primary school (Geary 2011).

\section{Capturing numerical quantities - subitizing, unstructured quantities, structured quantities - and their relevance for arithmetic}

Previous research indicated that already infants are sensitive to the differentiation of small quantities of 1 to 3 (e.g., Wynn 1992b; Antell, Keating 1983; Starkey 1992; Starkey et al. 1990; Feigenson et al. 2002). This ability has been associated with a specific system for the fast and more or less automatic processing of small quantities up to 3 or 4 referred to as subitizing (e.g., Kaufmann et al. 1949; Mandler, Shebo, 1982). Subitizing allows for grasping quantity information of small sets of 1 to 3 or 4 elements "at a glance" (Trick, Pylyshyn 1994; Clements, Sarama 2009). For young children in first grade there is also evidence for a so-called subitizing range of up to 3 elements (Trick et al. 1996).

Quantities beyond the subitizing range, starting with quantities 4 or 5 are assumed to be enumerated serially with more effort primarily relying on counting (e.g., Wender, Rothkegel 2000; Ashkenazi et al. 2013). This serial counting-based processing is associated with longer reaction times and more errors for increasing quantities in the so-called counting range (e.g., Plaisier et al. 2009). When children learn to count, they acquire the ability to enumerate larger quantities accordingly (e.g., Fuson, 1988; Ashkenazi et al. 2013).

A specific kind of enumerating quantity without subitizing or serial counting is found for structured quantities. Structured quantities are quantities which are always presented complying to distinct systematic patterns. Previous research indicated that recognizing patterns and structures is a central component of mathematical abilities (e.g., Lüken, Kampann 2018; Mulligan, Mitchelmore 2009). For instance, it was found for structured quantities that performance not necessarily decreases with increasing magnitude of the to-be-enumerated quantities (e.g., Wolters et al. 1987). These authors observed that accuracy and speed of enumerating quantities depended on the familiarity with structured quantities (Wolters et al. 1987). Mandler and Shebo (1982) postulated the canonical pattern hypothesis stating that canonical patterns, this means structured quantities reflecting systematic patterns one is familiar with and which are common in our culture, are enumerated faster and more accurately than unstructured 
quantities. Accordingly, it is assumed that children associate specific structured quantities with cardinality information in an iconic way.

There are different forms of structured quantities children are familiar with, with dice patterns being among the most prominent ones. Dice patterns usually represent magnitudes 1 to 6 in a canonical structured way. Results of Wender and Rothkegel (2000) supported the canonical pattern hypothesis of Mandler and Shebo (1982) with regard to dice patterns. The authors observed that dice patterns were processed faster than random sets of dots irrespective of their location on the screen or the size of the dots. Interestingly, Wender and Rothkegel (2000) also postulated that canonical patterns are processed in a holistic and iconic way like pictures. In this context, Dawson found already back in 1953 that structured quantities can be enumerated even better when they are organized in a regular and symmetric way, like dice patterns are (e.g. Bloechle et al. 2018; Krajcsi et al. 2013). In accordance with this evidence from adult participants, Benoit et al (2004) showed that children also enumerated quantities faster when presented in a structured was as dice patterns as compared to when presented in an unstructured way.

Due to their consistently structured representation of quantities, we assume that dice patterns do not only allow for grasping cardinality information but also for initial recognizing of some relationships between quantities. However, dice patterns only allow for specific relationships to be displayed. For instance, the dots reflecting six are arranged in two rows of three dots. As such dice patterns may allow children to recognize relationships between quantities in the sense that they convey specific decompositions of the to-be-reflected quantity in smaller quantities (e.g., two rows of three dots making six).

Another instantiation of such structured quantities are finger patterns as emerging from finger counting habits (e.g., thumb, index, and middle finger for 3). The overwhelming majority of children uses their fingers at some point during their numerical development to assist counting and/or initial calculations (Butterworth 1999a; Di Luca, Pesenti, 2011). Children use their fingers to gesturally present quantities (Fuson, Hall 1983) and they do so automatically and without specific instruction (Siegler, Shrager 1984). It is assumed that dealing with quantities by using ones' fingers (i.e., using fingers for counting, montring or manipulating quantities) facilitates learning and internalizing quantities through sensory-motor experiences (Di Luca, Pesenti 2011; see Roesch, Moeller, 2015 for a theoretical discussion), for instance, that more fingers raised represent a larger number (Wasner et al. 2014).

As such, finger-based variables have been found to have a critical influence on children's numerical development (e.g., Gracia-Bafalluy, Noël 2008). For example, performance in a finger discrimination task was found a good predictor for arithmetic performance in 5 to 8-year-old children (e.g., Fayol et al. 1998; Noël 2005). Moreover, it was observed that children who have problems in discriminating and naming their fingers performed worse in arithmetic tasks (Costa et al. 2011). Therefore, fingers are acknowledged as useful tools for the development of 
numerical abilities (Crollen, Noël 2015). Fingers support counting, help to track the count and even help to find solutions to arithmetic problems more easily because they allow for grasping the concept of composition and decomposition of numbers (cf. Roesch, Moeller, 2015, see also Butterworth, 1999).

In particular, it was argued that using fingers for dealing with quantities also leads to an iconic representation of the respective finger patterns in long term memory (Di Luca, Pesenti 2011). Domahs et al. (2008; 2010) observed that even when fingers are no longer used, internal traces of these external strategies affect number processing and arithmetic. In line with this, Di Luca and Pesenti (2011) postulated that finger-based numerical representations prevail even after symbolic numerical representations have developed. As such, finger counting and finger use for numerical operations more generally seems to shape and influence number processing and calculation throughout life (Di Luca, Pesenti, 2011; Domahs et al. 2010).

Based on such evidence, it was claimed that structured quantities like finger and dice patterns may facilitate the transition from concrete to abstract representations of number (Penner-Wilger et al. 2007) and support the ability to capture quantities (see also Butterworth, 1999, 2005) because they are processed in a more iconic way. In turn, this is assumed to help children refine their representation of quantity information, which should then facilitate understanding the principle of composition and decomposition of quantities as a precondition for early arithmetic abilities.

First evidence for this claim comes from both basic research on numerical cognition as well as mathematics education. As regards the former, Wijns and colleagues (2019) found that preschoolers that spontaneously produce patterns in a block assembly task were also better in math (see also Zippert et al., 2019; Rittle-Johnson et al, 2019, for similar results on the association of patterning skills and early arithmetic / mathematical abilities). Moreover, Burgoyne et al. (2019) observed that pattern understanding at the beginning of primary school was a significant longitudinal predictor of later arithmetic skills.

Moreover, the relevance of patterns and structures is also considered specifically in research on mathematics education (e.g., Sprenger, Benz, 2010). For instance, Lüken (2012) assessed how well children were able to consider patterns and structures by employing tasks requiring, amongst others, structuring objects in a way so that another person can see easily how many objects there are as well as identifying the number of elements in a set without counting them, three months before children started primary school. Results indicated that beyond influences of early numerical competences such as magnitude comparison, counting, and symbolic number knowledge - consideration of structures and patterns explained considerable additional variance in children's later mathematical abilities at the end of second grade. 
Importantly, patterning skills as assessed in these studies comprised recognition and use of predictable sequences (e.g., Zippert et al. 2019) on the one hand. On the other hand, they also drew on the ability to (quickly) recognize structures, as for example in cubes or in five or twenty-frames and so on (e.g., Lüken, 2012; Obersteiner et al. 2014). The latter may likely be based on structuring ideas used in instructional approaches in mathematics education (e.g., the power of five, Flexer, 1986; Krauthausen, 1995, or twenty-frames structured in sub-units of five, Obersteiner et al., 2014).

\section{The current study}

Based on these findings, the aim of the current study was to investigate whether mastery of structured quantities such as finger and dice patterns is relevant for the development of early arithmetic abilities in children in a longitudinal study from preschool to first grade. We assumed that structuring should help children to enumerate quantities in general and quantities beyond the subitizing range (i.e., larger than 3) in particular, when compared to unstructured quantities. Enumeration of unstructured quantities larger than 3 should primarily be done using rather time consuming and error prone counting procedures in preschool children (e.g., Schleifer, Landerl, 2011).

In contrast, structured quantities such as finger and dice patterns, which are familiar to children, may offer the possibility to enumerate quantities in an iconic way and thus faster and less error prone - in particular those larger than 3. We also assumed that children should benefit from the structuring of quantities as it provides some kind of external scaffold that helps them inferring the respective quantity information. This may allow them to handle the respective quantities more easily when asked to solve arithmetic operations. For instance children may consider the sub base 5 of their hands for tasks like $5+3$ (cf. Domahs et al. 2008) or they resort to the notion of dice patterns to solve the problem $3+3=6$ as the dice pattern for 6 consists of two rows of three dots each. Mastery of structured quantities may thus allow for a better as anchored understanding of quantities. As a consequence, we hypothesize that children's mastery of structured quantities should be associated specifically with their later arithmetic abilities: The better understanding of quantity which children establish based on structured quantities, should have a specific positive effect on their addition performance beyond that of their ability to enumerate unstructured quantities. To the best of our knowledge, there is no study evaluating potential differences between influences of finger vs. dice patterns as structured quantities compared with unstructured quantities (prominently solved by counting) and their specific prediction of later arithmetic performance. In particular, we investigated the association between mastery of quantities in preschool and arithmetic performance in first grade of primary school.

As such, this study aimed at i) replicating findings suggesting better enumeration of structured as compared to unstructured quantities before ii) evaluating the longitudinal relevance of performance in enumerating quantities, 
in particular of structured quantities, such as finger and/ or dice patterns in preschool for children's development of later arithmetic abilities in first grade.

\section{Method}

\section{Participants}

The present sample comprised 116 preschoolers $(N=58$ female) who took part in a school enrollment screening in Tyrol, Austria. Please note, the current manuscript focuses on results of only a subset of tasks administered in a larger longitudinal study conducted with the aim to develop a screening of language skills and basic numerical abilities at the transition from kindergarten to primary school. The tasks considered in this manuscript were administered to evaluate children's numerical development. All children were followed in a longitudinal study over the course of 7 months. In particular, they were first tested before starting primary school (first measurement time T1, from May to June) and again at the middle of first grade (second measurement time T2, from January to February of the following year). At T1 children were about six years old $(M=73.5$ months, $S D=3.64$, range 67 to 83 months) and on the second time of measurement (T2) they were about six years and a half $(M=80.5$ months, $S D=3.6$, range 74 to 90 months). The current study was approved by school authorities of the state of Tyrol and by the Research Committee for Scientific Ethical Questions (RCSEQ) of the UMIT - Private University for Health Sciences, Medical Informatics and Technology.

\section{Procedure and testing material}

At both times of measurement, children were tested in a 10-minute one-on-one session each. Testing itself was computer-based and done by trained student assistants who got detailed instructions of the procedure in advance. At T1 computer-based tests assessed children's ability to enumerate unstructured quantities (supposedly through counting), as well as structured quantities in terms of finger and dice patterns. At T2 an addition task was conducted. Computer tests were programmed with Experiment Builder (SR-Research, Ottawa, Canada) and measured reaction times as well as error rates. Computers used for testing were the same type for all children with a 16 ' screen.

\section{Unstructured quantities - Counting patterns}

The children's ability to tell the magnitude of unstructured quantities, was measured using 20 items (i.e., 2 practice trials, 18 critical unstructured quantities ranging from one to six, with each quantity presented 3 times), which were presented in randomized order. The task demanded children to identify the given quantity as quickly as possible and tell the respective number to the investigator, who recorded the respective answer. Unstructured quantities were shown for a maximum of 2 minutes when no key press has been received. There was an 
interstimulus interval of $500 \mathrm{~ms}$ when no answer was given before the next trial was presented. To increase children's motivation for the unstructured quantities the patterns were presented as pictures of dogs (see figure 1).

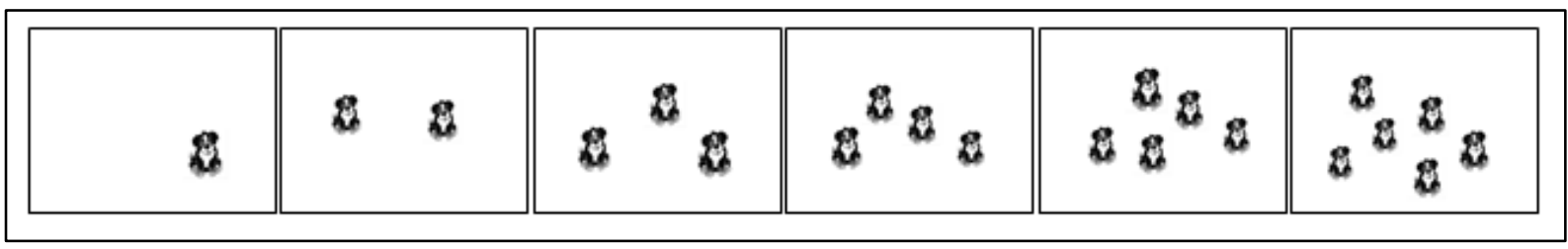

Fig 1 Examples of unstructured quantities ranging from 1 to 6 as used in the current study

\section{Structured quantities}

Children were also asked to enumerate the quantity given by structured quantities in terms of finger and dice patterns. Overall task procedure was identical to the one described for unstructured quantities above. In the following, specificities for the task on finger patterns and dice patterns will be described.

\section{Finger-patterns}

Finger-patterns were presented as photographs of children's hands in front of a background and depicted quantities from zero to ten because we anticipated enumeration of structured quantities to be easier. Following three practice items, each quantity was presented three times in randomized order (i.e., 3 practice trials, 30 critical trials). As for the task on unstructured quantities, individual items were presented for a maximum of 2 minutes or a response key was pressed within this interval. For quantities 1-6 we used the fingers thumb representing 1 , thumb and index finger reflecting 2, thumb, index, and middle finger indicating 3 , and so on according to the typical German finger counting habit (see figure 2). For reasons of comparability analyses will focus on results for quantities 1 to 6 in the following.

\section{Fingers}

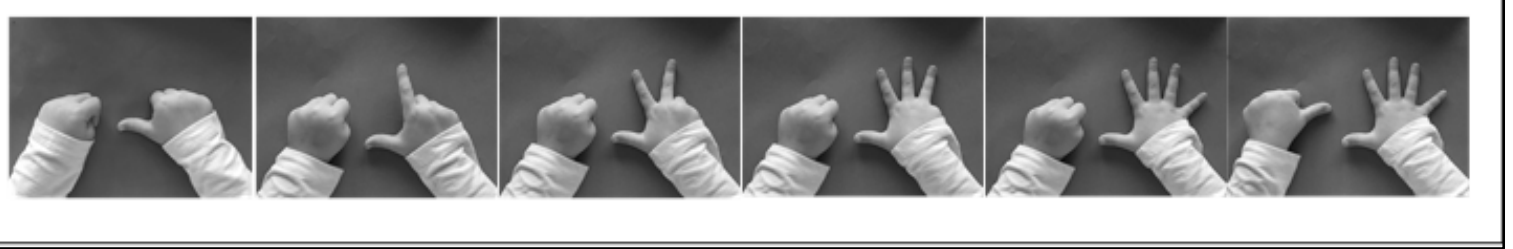

Fig 2 Finger patterns reflecting structured quantities as used in the present study

Due to the fact that children are not confronted with an unstructured representation of the fingerprints in everyday life, we refrained from using them in unstructured form. By unstructured form we mean finger pattern representations which are unusual in Europe (e.g., representation of 1 by the ring finger etc.).

\section{Dice-patterns}


The computer-based test evaluating children's ability to enumerate dice patterns involved 20 items presented in random order (i.e., 2 practice trials followed 18 critical trials presenting quantities from 1 to 6 three times each). Similar to the task on unstructured quantities, stimuli were presented until a key press was detected or the time limit of 2 minutes was reached. Additionally, dice patterns were also presented using pictures of dogs (see Figure 3) due to motivational reasons. The difference between the unstructured quantities to the dice patterns is that the dogs were arranged similar to dots on a dice (e.g., three dogs on a diagonal line representing the pattern of "three") (see figure 3).

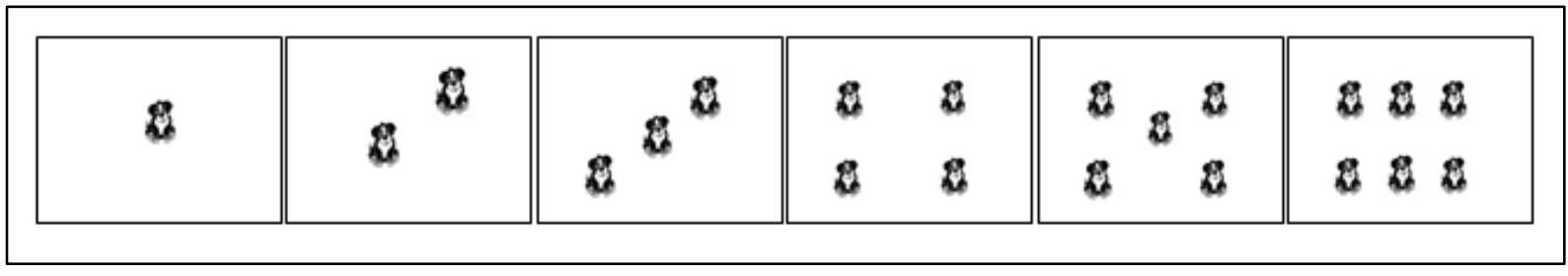

Fig 3 Structured quantities in terms of dice patterns as used in the current study

\section{Basic addition}

At T2 we conducted a test assessing children's ability to solve symbolic single digit additions which sums up to 10. The task demanded children to indicate as quickly and correctly as possible the result to a simple addition problem. We used a verification paradigm with two solution probes (i.e., the correct one and a wrong distractor differing from the correct results by plus or minus one, e.g., $4+1=5$ or 6 , see Figure 4) with items depicted on the computer screen. We chose a split of \pm 1 for incorrect solution probes (i.e., they differed from the correct solution by \pm 1 , e.g., $2+3=4$ vs. 5 vs. 6 ) based on previous evidence that the majority of incorrect solutions produced in addition come very close to the correct result (e.g. Ashcraft, Battaglia, 1978; for results in children see e.g., Domahs et al 2008; Núñez-Peña, Escera 2007).

For indicating the correct solution children had to press one of two response keys " $Z$ " and " $B$ " of a standard QWERTZ keyboard. When the upper solution probe was correct the upper button had to be pressed and vice versa for the lower solution probe and the lower response key. After a keystroke in the number field the next problem was presented. Problems were presented centered in white script against a black background in courier new bold, size 48 (see figure 4). Following 6 practice items, 40 critical items were presented in randomized order. After every 10 items children were able to take a short break for up to 4 minutes or until they pressed any key on the keyboard. 


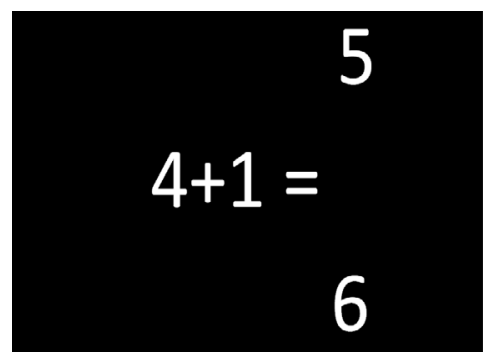

Fig 4 Example of stimuli used in the "addition test"

\section{Analyses}

Dice patterns as an instantiation of structured quantities only range from 1 to 6 . Therefore, we also restricted the range of structured quantities depicted as finger patterns to this range for the following analyses to allow direct comparability. As with the other computer tests, each quantity was presented three times, resulting in a total of 18 items. Data were analyzed using IBM SPSS Statistics version 24.

Analyses considered reaction times ${ }^{1}$ as well as the mean number of correct responses per quantity as dependent variables for all tests administered on the enumeration of unstructured and structured quantities (i.e., finger and dice patterns). As each quantity was presented 3 times the accuracy score could range from 0 (i.e., no item of any quantity solved correctly) to 3 (i.e., all items for all quantities solved correctly). For basic addition, the number of correctly solved problems was considered as dependent variable as reaction times were not registered for this task. Analyses of all 4 tasks will thus be based on the number of correctly solved items only (Table 1 presents the descriptive statistics for all variables).

Please note, because distributions of the mean number of correct responses were negatively skewed, we applied a $\log$ transformation on the reflected distribution of observed values to approximate normal distribution prior to the analyses. This resulted in skewness parameter that were within acceptable ranges (i.e., \pm 2 as previously suggested, e.g., Hair, Black, Babin, \& Anderson, 2010) for all variables of interest. Accordingly, for all results we report statistical details based on the analyses of transformed data. Please note, however, that we present untransformed data in all figures for reasons of comprehensibility.

Differences in median reaction time and the mean number of correct responses between enumerating unstructured quantities and structured quantities (i.e., finger and dice patterns) were evaluated by two separate 3 x 6 ANOVAs

\footnotetext{
${ }^{1}$ Please note, that due to technical problems (reaction times were not recorded on one out of three computers used for testing), reaction times were only available for a sub-sample of 90 children.
} 
discerning the within-participant factors presentation format (i.e., counting patterns vs. finger patterns vs. dice patterns) and quantities (i.e., 1, 2, 3, 4, 5, and 6).

In a next step, bivariate Pearson correlations were run to evaluate the association between enumeration performance for the three presentation formats as well later arithmetic performance.

Finally, a backward regression analyses was conducted to appraise the predictive relevance of enumeration performance for the three presentation formats for later arithmetic performance considering intercorrelations of predictors. Predictors were excluded from the regression model when not predicting the criterion variable with $p$ $<$.10. Please note that preliminary checks did not point to issues with multicollinearity as would have been indicated by correlations between predictors of $<.8$ in line with recommendations by Field (2018). Additionally, variance inflation factors for all variables included in the model were $<1.17$ (with VIF $>10$ indicating multicollinearity according to Kutner, 2005)

Table 1: Descriptive statistics for all test variables

\begin{tabular}{|c|c|c|c|c|c|}
\hline & $M$ & $S D$ & $N$ & Min. & Max. \\
\hline \multicolumn{6}{|l|}{ Preschool (T1) } \\
\hline Unstructured quantities & 2.69 & .41 & 116 & 0 & 3.00 \\
\hline Finger patterns & 2.84 & .35 & 116 & .50 & 3.00 \\
\hline Dice patterns & 2.92 & .29 & 116 & .17 & 3.00 \\
\hline \multicolumn{6}{|l|}{ First grade (T2) } \\
\hline Basic addition & 34.37 & 7.59 & 107 & 1.00 & 40.00 \\
\hline
\end{tabular}

\section{Results}

\section{ANOVA}

For the mean number of correct responses, the main effect of presentation format was significant $[F(2,115)=$ $\left.28.15, \eta_{p}{ }^{2}=.19, p<.001\right]$. Bonferroni-corrected pairwise comparisons indicated a significant difference between performance in enumerating unstructured quantities vs. dice patterns $(p<.001)$ as well as between performance in unstructured quantities and finger patterns $(p<.001$, see Figure 5). These results show that children enumerated structured quantities (i.e., finger and dice patterns) significantly better than unstructured quantities. Moreover, the pairwise comparison for finger and dice patterns also turned out to be significant $(p=.005)$. The latter reflected 
that enumeration performance was better for dice as compared to finger pattern indicating that children performed best on enumerating dice patterns.

The main effect of quantity also was significant $\left[F(5,115)=31.39, \eta_{p}{ }^{2}=.21, p<.001\right]$. Bonferroni-corrected pairwise comparison indicated significant performance differences between enumeration quantity 1 and 3 ( $p=$ $.038) 1$ and $4(p<.001), 1$ and $5(p<.001), 1$ and $6(p<.001)$ also between 2 and $4(p<.001), 2$ and $5(p<.001)$, 2 and $6(p<.001)$, as well as between 3 and $4(p<.001), 3$ and $5(p<.001), 3$ and $6(p<.001)$. In all cases, these results reflected that children enumerated smaller quantities with fewer errors than larger quantities (see figure 5).

Finally the interaction between presentation format and quantity was significant $\left[F(10,115)=19.70, \eta_{p}{ }^{2}=.15, p\right.$ $<.001]$. Bonferroni-corrected pairwise comparisons indicated that differences between presentation formats differed significantly across presented quantities.

Inspection of the simple effects indicated significant differences between the three presentation formats for the quantities 4,5 , and 6 , but not for quantities within the subitizing range (i.e., 1 to 3 ). For quantities 4 and 6 , fewer dice patterns were responded to significantly more accurately than unstructured quantities and dice patterns (both $p<.009)$. However, finger patterns were responded to less accurately than unstructured quantities for quantity 4 $(p<.003)$ but more accurately for quantity $6(\mathrm{p}<.001)$. Quantity 5 was enumerated significantly more accurately when presented as dice patterns $(p=.029)$ or finger patterns $(p=.028)$ as compared to unstructured quantities. Moreover, the pairwise comparison showed significantly better enumeration of quantity 5 for finger as compared to dice pattern $(p<.001)$. Overall, performance was lowest for unstructured quantities, whereas performance was best for dice patterns (see figure 5). Figure 5 shows the means and standard deviations of the number of correct responses for the quantities and the different presentation formats. 


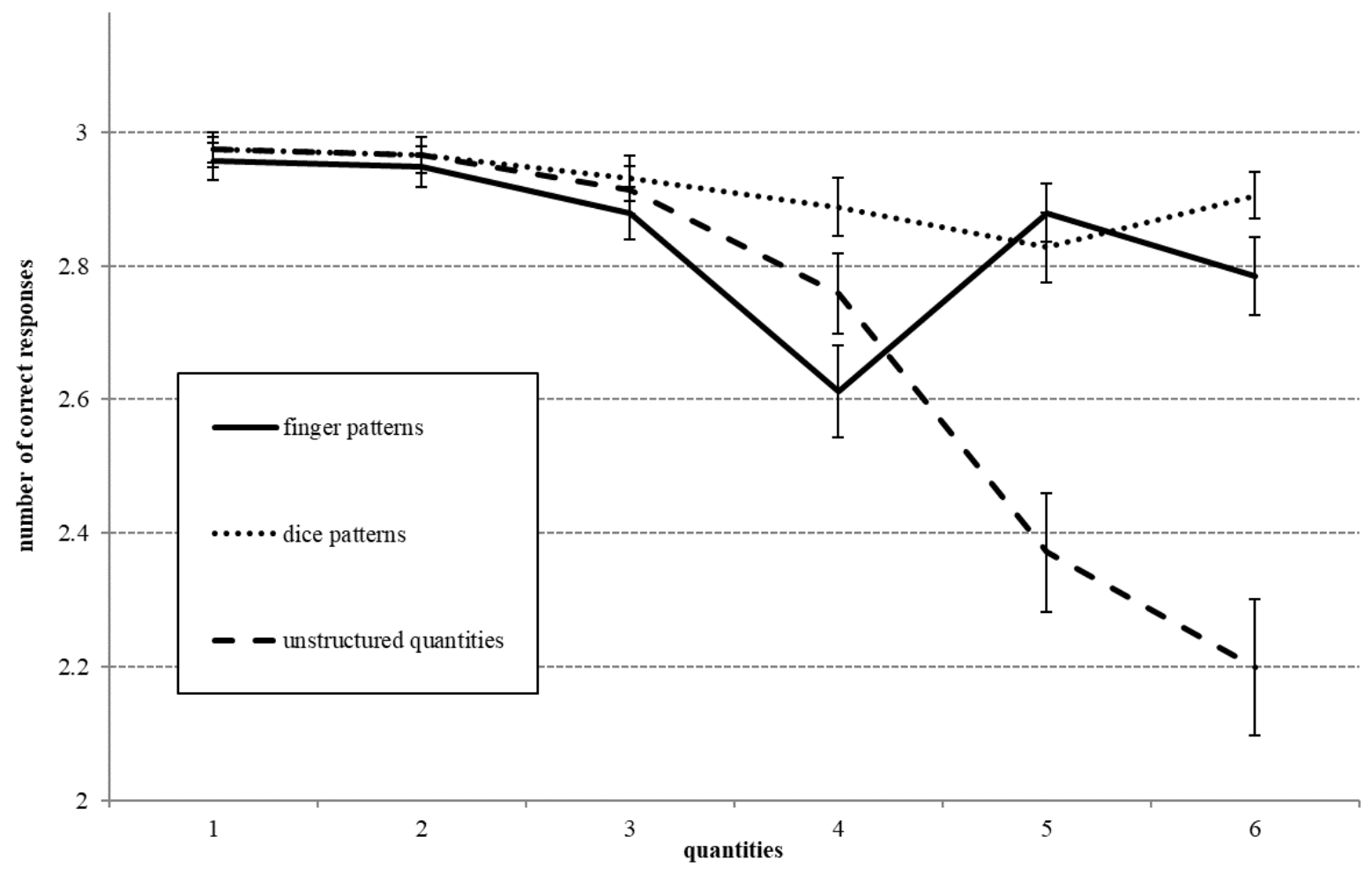

Fig 5 Mean number of correctly solved quantities separated for presentation format and quantity. Error bars indicate +/- 1 SEM

For median reaction times, the main effect of presentation format was significant $\left[F(2,89)=123.11, \eta_{p}{ }^{2}=.580, p\right.$ $<.001]$. Bonferroni-corrected pairwise comparisons indicated significant difference between reaction times for enumerating unstructured quantities and finger patterns $(p=.044)$, significant differences between reaction times for enumeration of unstructured quantities and dice patterns $(p<.001)$ as well as between enumerating finger patterns and dice patterns $(p<.001$, see Figure 6). These results show that children enumerated structured quantities which are arranged as dice patterns and finger patterns significantly faster than unstructured quantities but also dice significantly faster than finger patterns.

The main effect of quantity also was significant $\left[F(5,89)=114.74, \eta_{p}{ }^{2}=.563, p<.001\right]$. Bonferroni-corrected pairwise comparison indicated significant performance differences between the enumeration of all quantities (all $p<.001)$ except quantities 1 vs. $2(p>.99)$ and 4 vs. $5(p>.99)$.

Moreover the interaction between presentation format and quantity was significant $\left[F(10,89)=42.79, \eta_{p}^{2}=.325\right.$, $p<.001]$. Bonferroni-corrected pairwise comparisons indicated that differences between presentation formats differed significantly across presented quantities.

Inspection of the simple effects indicated significant differences for the reaction times between the three presentation formats for quantities 4,5 , and 6 , but not for quantities 1,2, and 3 (within the subitizing range). For quantity 4 , there was no significant difference between RT for enumerating unstructured quantities and finger 
patterns $(p>.383)$. However, pairwise comparisons between finger patterns and dice patterns as well as dice patterns and unstructured quantities showed significant RT advantages for dice patterns (both $p<.001$ ). For quantities 5 and 6 all pairwise comparisons were significant (all $p<.001$ ) reflecting that dice patterns were responded to fastest followed by finger patterns and unstructured quantities. Regarding quantity 6, all pairwise comparisons were significant (all $p<.001$ ) again indicating that dice patterns were responded to significantly faster than finger patterns which were responded to significantly faster than unstructured quantities. Taken together, it seems that the advantage for structured over unstructured quantities was primarily observed for quantities 5 and 6, for dice patterns also for quantity 4 (see figure 6).

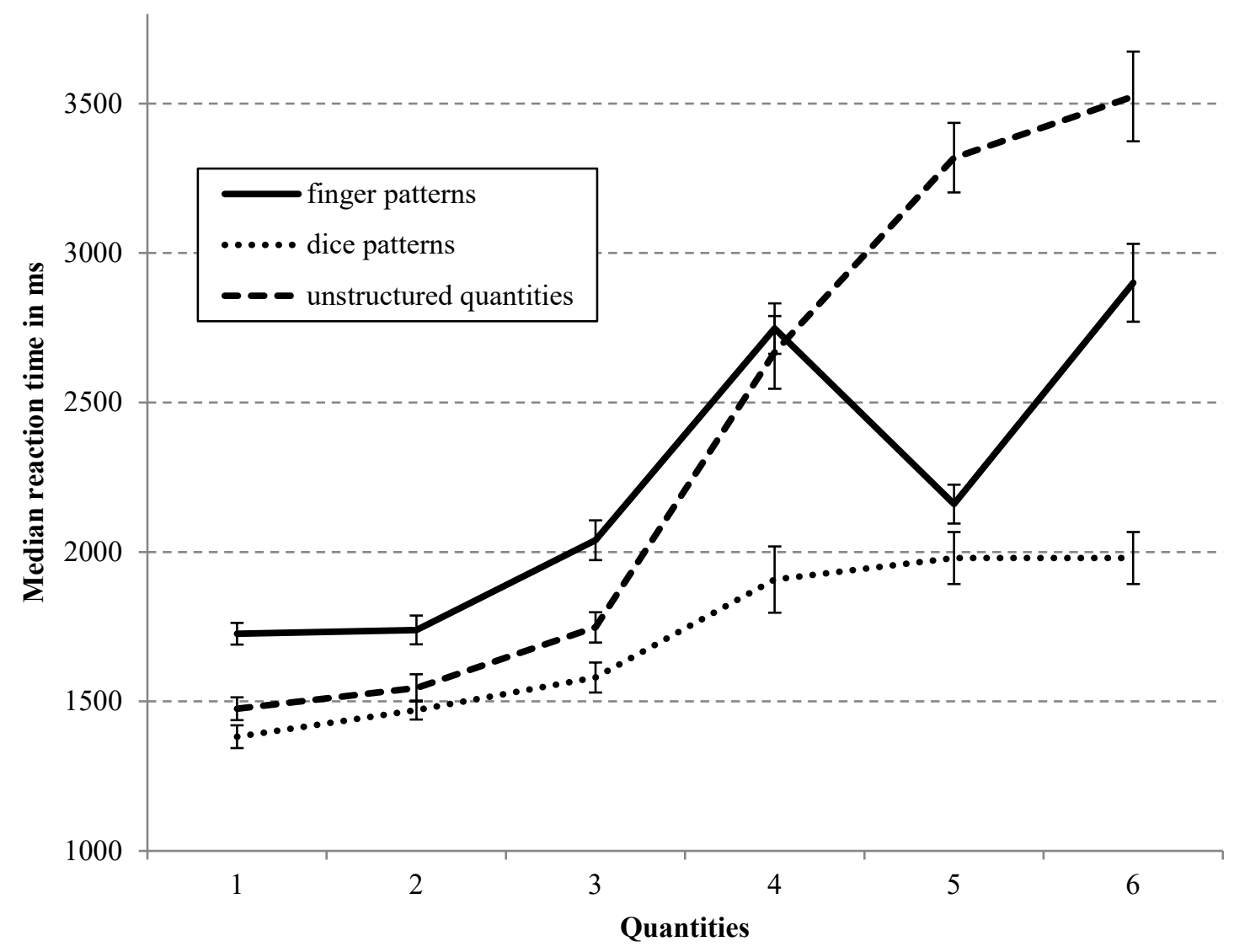

Fig 6 Median reaction times separated for presentation format and quantity. Error bars indicate $+/-1$ SEM

\section{Correlation analysis}

Table 2 depicts correlations between children's performance on quantity enumeration tasks and the basic addition. Table 2 shows that all tasks assessed correlated significantly with each other. In addition, relationships between the correlation of unstructured quantities and dice patterns as well as between finger and dice patterns are investigated. The lowest effect size showed the correlation of basic addition and unstructured quantities. 
Table 2: Correlations of the correct response of the tests (i.e., unstructured quantities, dice patterns, finger patterns, basic additions test)

\begin{tabular}{|c|c|c|c|c|}
\hline & & 1 & 2 & 3 \\
\hline 1 & Unstructured quantities (T1) & -- & & \\
\hline 2 & Dice patterns (T1) & $.317 * *$ & -- & \\
\hline 3 & Finger patterns (T1) & $.238 *$ & $.215^{*}$ & -- \\
\hline 4 & Basic additions (T2) & $.203 *$ & $.239 *$ & $.214^{*}$ \\
\hline
\end{tabular}

Note. ${ }^{*}$ The correlation is significant at the level of $\mathrm{p}<.05$ (2-sided)

\section{Regression analysis}

In the backward regression analysis, we evaluated whether the number of correct responses for the unstructured patterns as well as structured quantity enumeration tasks (i.e., finger \& dice patterns) assessed in preschool predicted children's performance in the addition test administered later in first grade.

The final model $\left[R^{2}=.08\right.$, adjusted $\left.R^{2}=.07, F(3,107)=4.69, p=.011\right]$ indicated that addition performance in first grade was predicted significantly by children's performance in the enumeration of dice patterns $(B=.219, S E$ $=.107, b=.19, p=.043)$. A significant prediction was also observed for enumeration of finger patterns $(B=.149$, $S E=.09, b=.17, p=.002)$. In contrast, enumeration of unstructured patterns was not a significant predictor of addition performance $(B=.097, S E=.09, b=.11, p=.269)$. Inspection of the beta weights indicated that better addition performance was predicted by better enumeration of dice and finger patterns. Enumeration of unstructured quantities did not seem to play a significant role for later addition performance. In fact, it can be assumed that this result indicates that counting as the most probable solution strategy for enumerating unstructured quantities no longer explained unique variance compared to the enumeration of structured quantities.

\section{Discussion}

The aim of this study was to evaluate i) differences between the processing of unstructured and structured quantities in preschool children and ii) influences of the mastery of structured quantities in preschool on children's arithmetic abilities in first grade. Consistent with our expectations, study results revealed that i) structured quantities were processed more efficiently than unstructured ones and ii) mastery of structured quantities (i.e., 
finger and in tendency also dice patterns) in preschool specifically predicted children's later arithmetic abilities. In the following, we will discuss these points in turn.

\section{Advantage for processing structured quantities}

In line with our expectation, it turned out that structured quantities were enumerated more efficiently than unstructured ones as indicated by the main effect presentation format we observed. In particular, performance was significantly better for enumerating both structured quantities (i.e., finger and dice patterns) as compared to unstructured quantities. This is in accordance with previous findings and theoretical claims suggesting that enumerating unstructured quantities (i.e. counting pattern) involves more effortful and time-consuming serial counting processes as compared to structured quantities (e.g., Ashkenazi et al., 2013). Arguing with Wolters et al. (1987) we found that children were able to process quantities better when they were presented in structured format, like finger or dice patterns - which also corresponds well with the canonical hypothesis by Mandler and Shebo (1982).

Importantly, the significant interaction between presentation format and the actual quantity to be enumerated indicated that above described processing benefit of structured quantities over unstructured ones was only present for quantities larger than three and thus outside the subitizing range. This indicates that for small quantities within the subitizing range there does not seem to be a specific benefit of structure for enumerating quantities. For quantities larger than 3 our data clearly indicate a processing benefit for structured quantities over unstructured ones for quantities 5 and 6 , for dice patterns also for quantity 4 . A closer look at the simple effects further specified the observation that performance for dice patterns was significantly better than for finger patterns, for which performance was better than for unstructured quantities to also be driven by the respective effects for quantities larger than 3 .

We not only observed that performance for both structured quantities (i.e., finger and dice patterns) was better than for unstructured quantities (for accuracy) but also that performance in enumerating dice patterns was better than for finger patterns (for accuracy and speed). The former provides converging evidence for ideas that see structured quantities as prominent maybe even iconic representations of numerical magnitude (e.g., Di Luca and Pesenti, 2011; Wender and Rothkegel, 2000) as it substantiates the processing advantage for structured quantities to be observed as early as kindergarten. As regards the latter, one might speculate that dice patterns are the proto-typical instance of structured quantities acquired through children's experiences with dice in different contexts such as playing board games and so on (cf. Wolters et al. 1987) - which might explain their processing advantage over finger patterns. Another argument for the even more efficient processing of dice patterns seems to be their symmetrical arrangement (cf. Dawson 1953) for which processing advantages were observed, probably influenced 
by processes of pattern recognition (c.f. Mandler \& Shebo, 1982; Krajcsi et al. 2013). As such, finger patterns may also be seen as a advantageous iconic representation of structured quantities in preschoolers (that might even influence number processing throughout life, e.g. Di Luca \& Pesenti, 2011; Domahs et al. 2010). On the other hand, finger patterns for quantities 1 to 6 as used in the current study are not symmetrical but follow a specific order of fingers raised one after the other. Interestingly, however, the (reversed) v-shaped increase of performance for the finger patterns for 5 (with significantly fewer errors committed for finger than for dice patterns) seems to indicate that for finger patterns representations of 5 as a full hand and thus a superior semantic category may have improved processing further.

Synced with the results for the main effect of quantity, which basically showed no difference for processing small quantities up to 3 , this clearly reflects subitizing of these small quantities - meaning fast and largely automatic processing of the respective quantities with no significant influences of presentation format. In contrast for quantities 4 to 6 there was a linear decrease of performance in line with previous results (e.g., Ashkenazi et al. 2013; Schleifer and Landerl 2011).

Interestingly, the finger pattern for 4 seemed particularly difficult to process. A possible reason for this might be that for 4 two finger patterns are prominently used (e.g., Wasner et al. 2016): i) according to the finger counting sequence, this means thumb, index, middle, and ring finger vs. a motorically easier one involving index, middle, and ring finger as well as the pinkie. This may have made the finger pattern for 4 less beneficial. Additionally, we also observed a special status for 5 . As observed previously (e.g. Crollen \& Noel, 2015) the finger pattern for 5 was enumerated specifically fast and accurate. This may reflect that 5 is conveyed by one whole hand, which adds further structural semantic meaning beyond the quantity information itself.

\section{Mastery of structured quantities predicts later arithmetic abilities}

As expected, we observed that mastery of structured quantities - finger patterns, and dice patterns - as assessed in preschool uniquely predicted later arithmetic abilities in first grade. This seems to indicate that mastery of finger as well as dice patterns seem to account for distinct parts of the variance in addition performance - even though it needs to be acknowledged that the final model explained only a small part of the variance. Furthermore, although the correlation between addition performance and enumeration of unstructured quantities was significant, the latter was not a significant predictor of addition performance in the regression analysis. This may indicate that enumeration of unstructured quantities did not account for unique parts of variance, but may share parts of variance with the mastery of structured quantities. This argument is backed by significant correlation between enumeration of unstructured quantities and structured quantities (finger and dice patterns). 
From a content-wise perspective, this may reflect that children can already count before they start to specifically consider structured quantities. As such, counting may be a prerequisite for the later consideration of structured quantities and thus, no longer explains unique parts of the variance of addition performance. Interestingly, we found not only mastery of dice patterns but also mastery of finger patterns to be - in tendency - predictive of later arithmetic performance. This further strengthens theoretical claims (e.g., Butterworth 1999; 2005, Moeller, Roesch 2015) postulating beneficial effects of finger-based numerical representations on children's numerical development. In particular, when children use their fingers for counting and/or initial calculations, they explicitly operate on quantities and raising another finger or closing a finger already allows for acquiring first conceptual ideas on increasing and decreasing quantities and thus first arithmetic procedures through the use of their fingers.

In addition to the results of earlier research (e.g. Noël 2005; Penner-Wilger et al. 2007), which investigated the influence of fine motor skills and finger gnosis on early numerical and calculation skills, the present results indicate that the explicit use of finger patterns might be beneficial for later arithmetic performance. In particular, these results imply that not only the concrete acting of counting/calculating with one's fingers but also the therewith acquired iconic representation of finger patterns influences later arithmetic performance positively - at least in tendency.

Not only fingers (see Penner-Wilger et al. 2007) may facilitate transition from concrete to more abstract numerical representations, but also dice patterns may be beneficial for this transition, as reflected in our data. This could be seen for the mastery of dice patterns to predict later arithmetic performance. As such, dealing with dice should have a beneficial influence on later arithmetic performance. This is in line with results from a study by Gasteiger, Obersteiner and Reiss (2015) who observed that children benefitted more form playing board games with a regular dot dice than playing board games with colour or symbol dice. In particular, the authors found that children improved in their ability to consider structures when dealing with sets of objects.

Taken together, we suggest that structured quantities such as finger and dice patterns allow children better capture quantity information because of the systematic and spatially structured nature of these formats which facilitate understanding of quantities as composed and to-be-decomposed units. This, in turn, seems to benefit children's early arithmetic abilities - possibly because it already represents pre-arithmetic operations. Moreover, the iconic representation of structured quantities may enable more efficient access to quantity information which should also improve arithmetic abilities.

Nevertheless, a point to consider when interpreting these results is that we did not control for influences of domainunspecific variables known to impact on numerical development such as general cognitive abilities, working memory, etc. (e.g. Raghubar et al. 2010; Cowan et al. 2017 for influences of these variable). However, we observed 
a pattern of specific associations of structured quantity enumeration and later arithmetic performance, while at the same time enumeration of unstructured and structured quantities was correlated significantly. This pattern of results clearly argues against confounding influences of domains-unspecific variables.

As a further limitation, it needs to be noted that we cannot completely rule out the possibility that children also resorted to counting-based strategies when solving the addition task in the current study. However, there is one specific to our results that clearly argues against the assumption that the addition task was solely solved using counting-based strategies. Following the latter argument, one would expect a particularly high association of performance on the enumeration of unstructured quantities task, which we expect to be solved by counting and the addition task, which one might assume to also be solved by counting-based strategies. However, our results clearly indicate that performance in the addition task was better predicted by performance in the enumeration of structured quantities task, which is not primarily solved by counting but by specifically considering quantity information as conveyed by structured quantities. Therefore, we are confident that the addition task we used reflected more than just counting based strategies.

Finally, it needs to be noted that we did not consider the theoretically and conceptually aspect of number composition and decomposition (e.g., Sarama \& Clements 2009) to be pursued in the current study. As such future research should pay more attention on conceptualizing finger as well as dice patterns as composed units allowing to foster children's acquisition of number composition and decomposition (e.g., six as displayed in two rows of three dots on a dice or possibilities of decomposing ten fingers two numbers adding up to ten, see also Roesch and Moeller, 2015 for a discussion on how finger-based strategies can foster the acquisition of initial calculations).

\section{Conclusions}

The results of the current study indicate that children can achieve a better understanding of quantities by dealing with structured quantities, which in turn seems to have a positive influence on later arithmetic performance. In particular, we found that mastery of dice and also finger patterns predicted later addition performance. Interestingly, the role of dice patterns as structured quantities seemed to be more prominent as compared to finger patterns, which might be due to the more extensive experience of children with dice patterns in, for instance, board games and their symmetrical arrangement. In summary, this suggests that it may be beneficial to instruct children more systematically on structured quantities as their specific association and prediction of later arithmetic performance structured quantities may foster children's development of more complex arithmetic abilities. 


\section{Acknowledgements}

We thank the participating schools and the students for their assistance with the data collection. Special thanks go to the participating children and their parents for their cooperation. We also thank the tyrolean science fond (TWF: Uni-0404-2101) for their support. The authors declare no competing interests.

\section{Disclosure statement}

There are no conflicts of interests to declare.

Ethical approval: All procedures performed in this study were in accordance with the ethical standards of the ethics committee of the UMIT - Private University for Health Sciences, Medical Informatics and Technology $\mathrm{GmbH}$.

Informed consent: Informed consent was obtained from all individual participants included in the study and their parents or legal guardians.

\section{References}

Antell SE, Keating DP (1983) Perception of numerical invariance in neonates. Child Development 54: 695-701

Ashkenazi S, Mark-Zigdon N, Henik A (2013) Do subitizing deficits in developmental dyscalculia involve pattern recognition weakness? Developmental Science 16: 35-46

Ashkraft M H, Battaglia J (1978) Cognitive Arithmetic: Evidence for Retrieval and Decision Processes in Mental Addition. Journal of Experimental Psychology Human Learning and Memory 4: 527-538

Benoit L, Lehalle H, Jouen F (2004) Do young children acquire number words through subitizing or counting? Cognitive Development 19: 291-307

Bishop JH (1989) Is the test score decline responsible for the productivity growth decline? American Economic Review 79: 178-197

Bloechle J, Huber JF, Klein E, Bahnmüller J, Rennig J, Moeller K., Huber S (2018) Spatial Arrangement and Set Size Influence the Coding of Non-symbolic Quantities in the Intraparietal Sulcus. Frontiers in Psychology https://doi:10.3389/fnhum.2018.00054

Burgoyne K, Malone S, Lervag A, Hulme C (2019) Pattern understanding is a predictor of early reading and arithmetic skills. Early Childhood Research Quarterly 49: 69-80 
Butterworth B (1999) The mathematical brain. Macmillian, London

Butterworth B (1999a) A head for figures. Science 284: 928-929

Butterworth B (2005) The development of arithmetical abilities. Journal of Child Psychology and Psychiatry 46: 3-18

Clements D H, Sarama J (2009) Learning and Teaching Early Math. Routledge, New York

Costa AJ, Silva JBL, Chagas PP, Krinzinger H, Lonneman J, Willmes K., Wood G, Haase VG (2011) A hand full of numbers: a role for offloading in arithmetics learning? Frontiers in Psychology. https://doi.org/10.3389/fpsyg.2011.00368

Cowan R, Hurry J, Midouhas E (2017) The relationship between learning mathematics and general cognitive ability in primary school. Developmental Psychology https//doi: 10.1111/bjdp.12200

Crollen V, Noël MP (2015) The role of fingers in the development of counting and arithmetic skills. Journal of Educational Psychology 90: 321-329

Dawson D T (1953) Number grouping as a function of complexity. Elementary school journal 54: 35-42

Di Luca S. Pesenti, M (2011) Finger Numeral Represenations: More than Just Another Symbolic Code. Frontiers in Psychology 2: 272

Domahs E, Krinzinger H, Willmes K (2008) Mind the gap between both hands: evidence for internal finger-based number representations in children's mental calculation. Cortex 44: 359-367

Domahs E, Moeller K, Huber S, Wilmes K, Nuerk H C (2010) Embodied numerosity: Implicit hand-based representations influence symbolic number processing crosses cultures. Cognition 116: 251-266

Fayol M, Barrouillet P, Marinthe C (1998) Predicting arithmetical achievement from neuropsychological performance: a longitudinal study. Cognition 68: 63-70

Feigenson L, Carey S, Hauser MD (2002) The representation underlying infant's choice of more: object file vs. Analog magnitudes. Psychological Science 13: 150-156

Field A P (2018). Discovering statistics using IBM SPSS statistics. London, Thousand Oaks

Flexer R J (1986) The Power of Five: The Step before the Power of Ten. Arithmetic Teacher 34: 5-9 
Fuson K.C, Hall JW (1983) The Acquisition of Early Number Word Meanings: A Conceptual Analysis and Review. In Ginsburg HP (ed) The Development of Mathematical Thinking. Academic Press, London, pp 49107

Fuson K.C (1988) Children's counting and concept of number. Springer, Heidelberg

Gasteiger H, Obersteiner A, Reiss K (2015) Formal and informal learning environments: Using games to support early numeracy. In Torbeyns J, Lehtinen E, Elen J (ed) Describing and Studying Domain-Specific Serious Games. Springer, Cham, pp 231-250

Geary D (2000) From infancy to adulthood: the development of numerical abilities. European Child \& Adolescent Psychiatry 9: 11-16

Geary D C (2011) Cognitive predictors of achievement growth in mathematics: A 5-year longitudinal study. Developmental Psychology 47: 1539-1552

Gracia-Bafalluy M, Noël MP (2008) Does finger training increase young children's numerical performance? Cortex 44: 368-375

Hair J, Black W C, Babin B J, Anderson R E (2010) Multivariate data analysis ( $7^{\text {th }}$ ed.) Upper Saddle River, New Jersey: Pearson Educational International

Hirsch S, Lambert K., Koppens K, Moeller K (2018) Basic numerical competences in large-scale assessment data: Structure and long-term relevance. Journal of Experimental Child Psychology 167: 32-48

Kaufmann EL, Lord MW, Reese TW, Volkmann J (1949). The discrimination of visual number. American Journal of Psychology 62: 498-525

Krajcsi S, Szabó E, Móroz IÁ (2013) Subitizing is sensitive to the arrangement of objects. Experimental Psychology 60: 227-234

Krauthausen G (1995) Die „Kraft der Fünf“ und das denkende Rechnen. Zur Bedeutung tragfähiger Vorstellungsbilder im mathematischen Anfangsunterricht. In Müller G N, Wittman E C (ed) Mit Kindern rechnen. Arbeitskreis Grundschule-Der Grundschulverband-eV Frankfurt am Main, pp. 87-108

Kutner M H (2005) Applied linear statistical models. Boston, McGraw-Hill Irwin

Lüken M (2012) Young Children's Strcture Sense. Journal für Mathematik-Didaktik 33: 263-285 
Lüken M Kampmann R (2018) The influence of fostering children's pattern and structure abilities on their arithmetic skills in Grade 1. In Elia I Mulligan J Anderson A Baccaglini-Frank A \& Benz C (ed), Contemporary research and perspectives on early childhood mathematics education. Springer, Heidelberg, pp 55-69

Mandler D, Shebo BJ (1982) Subitizing: an analysis of its component processes. Journal of Experimental Psychology: General 111: 1-22

Mulligan J, Mitchelmore M (2009) Awareness of Pattern and Structured in Early Mathematical Development. Mathematics Educations Research Journal 21: 33-49

Noël MP (2005) Finger gnosia: A predictor of numerical abilities in children? Child Neuropsychology 11: 413430

Núñez-Peña I, Escera M (2007) An event-related brain potential study of the arithmetic split effect. International Journal of Psychophysiology 64: 165-173

Obersteiner A, Reiss K, Ufer S, Luwel K, Verschaffel L (2014) Do first graders make efficient use of external number representations? The case of the twenty-frame. Cognition and instruction32: 353-373

Penner-Wilger M, Fast L, LaFevre J, Smith-Chant BL, Skwarchuck S, Kamawar D, Bisanz J (2007) The Foundations of Numeracy: Subitizing, Finger Gnosia, and Fine Motor Ability. Proceedings of the Annual Meeting of the Cognitive Science Society, 29. Retrieved from https://escholarship.org/uc/item/8vb45554

Pixner S, Kraut C, Dresen V (2017) Early Predictors for Basic Numerical and Magnitude Competencies in Preschool Children - Are They the Same or Different regarding Specific Subgroups? Psychology 8: 271286

Plaisier MA, Bergmann WM, Kappers AML (2009) One, two, three, many - Subitizing in active touch. Acta Psychologica 131: 163-170

Raghubar KP, Barnes M, Hecht S (2010) Working memory and mathematics: A Review of developmental, individual difference and cognitive approach. Learning and Individual Differences 20: 110 - 122

Rittle-Johnson B, Zipper E L, Boice K L (2019) The roles of patterning and spatial skills in early mathematics development. Early Childhood Research Quarterly 46: 166-178

Rivera-Batiz FL (1992) Quantitative literacy and the likelihood of employment among young adults in the United States. Journal of Human Resources 27: 313-328 
Roesch S, Moeller K (2015) Considering digits in a current mode of numerical development. Frontiers in human neuroscience 8: 1062

Sarama J, Clements D H (2009) Early childhood mathematics education research: Learning trajectories for young children. Routledge, Abingdon

Schleifer P, Landerl K (2011) Subitizing and counting in typical and atypical development. Developmental Science 14: $280-291$

Siegler RS, Booth JL (2004) Development of Numerical Estimation in Young Children. Child Development 75 : $428-444$

Siegler RS, Shrager J (1984) Strategy choices in addition and subtraction: How do children know what to do? In Sophian C (ed) Origins of Cognitive Skills. The Eighteenth Carnegie Symposium on cognition. Erlbaum, Hillsdale, pp 229-293

Sprenger P, Benz C (2010) Children's perception of structures when determining cardinality of sets - results of an eye-tracking study with 5-year-old children. https://doi.org/10.1007/s11858-020-01137-x

Starr A, Libertus ME, Brannon EM (2013) Number Sense in Infancy Predicts Mathematical Abilities in Childhood. Proceeding of the National Academy of Sciences, 110: 18116-18120

Starkey P (1992) The early development of numerical reasoning. Cognition 43: 313-328

Starkey P, Spelke ES, Gelman R (1990) Numerical abstraction by human infants. Cognition 36: 97-127

Trick LM, Pylyshyn ZW (1994) Why are small and large numbers enumerated differently? A limited capacity preattentive stage in vision. Psychological Review 101: 80-102

Trick LM, Enns JT, Brodeur DA (1996) Life span changes in visual enumeration: The number discrimination task. Developmental Psychology 32: 925-932

Wasner M, Moeller K, Fischer MH, Nuerk HC (2014) Aspects of situated cognition in embodied numerosity: The case of finger counting. Cognitive Processing 15: 317-328

Wender KF, Rothkegel R (2000). Subitizing and its subprocesses. Psychological Research 64: 81-92

Wijns N, De Smedt B. Verschaffel L, Torbeyns J (2019) Are preschoolers who spontaneously create patterns better in mathematics?. British Journal of Educational Psychology. 
Wolters G, van Kempen H, Wijlhuizen GJ (1987) Quantification of small numbers of dots: Subitizing or pattern recognition? American Journal of Psychology 100: 225-237

Wynn K (1992b) Children's acquisition of the number words and the counting system. Cognitive Psychology 24 : 220-251

Zippert E. Douglas A A, Smith M R, Rittle-Johnson B (2019) Preschoolers' broad mathematics experience with parents during play. Journal of Experimental Child Psychology192 https://doi:10.1016/j.jecp.2019.104757 